\title{
In situ repair of vagus nerve stimulator lead damage: technical note
}

\author{
Ashley Ralston, MD, ${ }^{1}$ Patti Ogden, APN, ${ }^{2}$ Michael H. Kohrman, MS, MD, ${ }^{2}$ and \\ David M. Frim, MD, PhD'
}

Sections of ${ }^{1}$ Neurosurgery and ${ }^{2}$ Pediatric Neurology, The University of Chicago, Illinois

\begin{abstract}
Vagus nerve stimulators (VNSs) are currently an accepted treatment for intractable epilepsy not amenable to ablative surgery. Battery death and lead damage are the main reasons for reoperation in patients with VNSs. In general, any damage to the lead requires revision surgery to remove the helical electrodes from the vagus nerve and replace the electrode array and wire. The electrodes are typically scarred and difficult to remove from the vagus nerve without injury. The authors describe 6 patients with VNSs who presented with low lead impedance on diagnostic testing, leading to the intraoperative finding of lead insulation disruption, or who were found incidentally at the time of implantable pulse generator battery replacement to have a tear in the outer insulation of the electrode wire. Instead of replacement, the wire insulation was repaired and reinforced in situ, leading to normal impedance testing. All 6 devices remained functional over a follow-up period of up to 87 months, with 2 of the 6 patients having a relatively shorter follow-up of only 12 months. This technique, applicable in a subset of patients with VNSs requiring lead exploration, obviates the need for lead replacement with its attendant risks.
\end{abstract}

http://thejns.org/doi/abs/10.3171/2016.6.PEDS169

KEY WORDS vagus nerve stimulator; electrode repair; epilepsy; technique

$\mathrm{S}$ URGICALLY implanted vagus nerve stimulators (VNSs) have been used for the treatment of refractory epilepsy since the first VNS was implanted in a human in 1988 and subsequent US FDA approval in $1997 .{ }^{2}$ End of battery life and lead damage are the main reasons for reoperation in these patients. Lead damage can be detected by the finding of low lead impedance or lead fracture radiographically. Historically, when a lead is damaged, a revision is required to remove the electrode array and wires and place a new electrode array on the nerve with the attendant risks of dissecting the carotid artery and internal jugular vein. Some surgeons prefer to avoid the mass of scar containing the nerve, electrodes, and vessels by enlarging the incision, leaving the damaged electrodes in place, and placing new leads on a virgin area of nerve. To our knowledge, there are no reported techniques describing in situ repair of VNS leads. We present an alternative technique, applicable to leads with insulation damage and without obvious lead disconnection, which allows repair, without replacement, of the electrode array.

\section{Methods}

This is a retrospective review of 6 patients treated for malfunction of their VNS leads with repair, instead of replacement (Table 1). They were under the care of a pediatric neurosurgeon and pediatric epileptologist at the University of Chicago Medical Center, and all patients were included in an institutional review board-approved study. All patients tolerated the procedure well and were discharged home the same day. Of the 6 patients, 5 presented with low lead impedance when interrogated preoperatively. One patient had normal impedance testing, but was found during the course of an elective implantable pulse generator (IPG) battery replacement to have fractured electrode insulation and underwent repair prophylactically. Postrepair lead impedance was in the normal range, and no side effects or complications were observed due to the repair or the materials used.

The concept of lead repair was formulated prior to performing the first case, which is reported. Low lead 
TABLE 1. Patient characteristics

\begin{tabular}{cclclc}
\hline $\begin{array}{c}\text { Case } \\
\text { No. }\end{array}$ & $\begin{array}{c}\text { Age (yrs), } \\
\text { Sex }\end{array}$ & \multicolumn{1}{c}{ Medical History } & Revision (mos) & Reason for Revision & Findings \\
\hline 1 & $17, \mathrm{~F}$ & Epilepsy, TS & 75 & Increased seizures \& presumed battery death & Frayed outer sheath \\
\hline 2 & $10, \mathrm{~F}$ & Epilepsy; chromosome 6,21 translocation & 79 & Presumed battery death & Frayed outer sheath \\
\hline 3 & $17, \mathrm{~F}$ & Epilepsy; chromosome 6,21 translocation & 79 & Low impedance & Frayed outer sheath \\
\hline 4 & $12, \mathrm{~F}$ & Epilepsy, TS & 87 & Increased seizures \& low impedance & $\begin{array}{r}\text { Frayed outer sheath \& } \\
\text { exposed inner wires }\end{array}$ \\
\hline 5 & $21, \mathrm{~F}$ & Epilepsy, TS & 139 & Increased seizures \& presumed battery death & Frayed outer sheath \\
\hline 6 & $31, \mathrm{~F}$ & Epilepsy & 126 & Low lead impedance & Frayed outer sheath \\
\hline
\end{tabular}

TS = tuberous sclerosis.

impedance was demonstrated preoperatively, and chest radiography did not reveal any visible disconnections or disruptions in the radiopaque electrode wire. Preoperative discussion with the family centered around the possible need for replacement of the leads. However, with the electrode wires in continuity, the possibility of disruption of the insulation was also reviewed with the family. As an alternative to lead replacement, repair of the damaged insulation was offered as described below by using Silastic tubing, commonly implanted for CSF shunting. The use of the catheter was intended to provide support around an area of damaged insulation, presumably from the stress of bending within the pocket. The addition of cyanoacrylate glue was used to restore the integrity of the insulation within the small opening where the catheter had been split to allow placement around the electrode.

In review of VNS procedures performed at the University of Chicago from January 2012 through May 2015, 15 patients, all male, required lead replacement. Three of our reported lead repairs were performed during this time. Of the 15 lead replacements, 10 were due to lead fractures or disconnections (Fig. 1) and demonstrated high lead impedance $(9000-10,000 \Omega$ ) on preoperative testing. The 5 remaining cases exhibited elevated impedance $(>10,000$ $\Omega$ ) without evidence of fracture. This finding is in contrast to our patients, who had low impedance. An interesting observation is that there were no male patients included in our cohort. One theory is that young male patients tend to be more active, leading to electrode damage beyond the insulation, requiring complete lead replacement.

\section{Repair Technique}

Preoperative low lead impedance heightened the likelihood of lead damage; however, in all cases, careful scrutiny was used to inspect the entirety of the coiled electrode leads exposed in the subcutaneous pocket during expected revision. The likelihood of missing other areas of lead damage was felt to be low, as most damage occurs in areas where the lead is coiled within the pocket. Electrical reevaluation of the system after repair demonstrated improved lead impedance, which would not be expected if there were unseen areas of damage. When the lead insulation was noted to be compromised, the electrode was irrigated with antibiotic-containing solution (Bacitracin) and dried with a gauze sponge. We then measured an appropriate length of Silastic tubing (Holter catheter, Codman/ Johnson \& Johnson) that was sufficient to cover the dam- aged outer sheath with an additional $1-2 \mathrm{~cm}$ of tubing extending beyond the damage on either side. A fresh No. 11 surgical scalpel was used to make a lengthwise cut down one side of the catheter tubing, opening the catheter so it could be slipped over the damaged area. The cut catheter was then delicately placed around the damaged sheath and secured at multiple points with circumferential 2-0 silk ties until there was no longer a bend in the lead or exposed inner electrode wires. Cyanoacrylate glue (Dermabond, Ethicon/Johnson \& Johnson) was then introduced into the open side of the cut catheter as a sealant in and around the damaged external insulation, up to either end of the repair. The glue was then allowed to fully dry. At this point, the repaired segment was handled in a similar fashion to the undamaged wiring and reimplanted.

In one of the patients (Case 4 in Tables 1 and 2), the internal wiring also appeared exposed due to damage to the internal insulation. In this case, the 2 damaged internally insulated wires were treated individually but in a similar fashion to the damaged external insulation. The internal wires were placed within a smaller caliber lumbar drainage catheter (Lumbar Drainage Catheter Kit II, Codman/Johnson \& Johnson) that had been slit along the side at length appropriate to reinforce the damaged internal insulation. After delicate placement within the lumbar drainage tubing, 2-0 silk ties were used to nearly close the tubing, and cyanoacrylate glue was applied to reinsulate and reinforce (Fig. 2). When dry, the 2 reinforced inner wires were treated as "damaged" and placed within sideslit tubing, secured with silk ties, and treated with another application of cyanoacrylate glue before reinsertion.

All 6 devices showed appropriate lead impedance after repair (Table 2), and these values remained stable once the battery was secured in the subcutaneous pocket and the skin incision was closed.

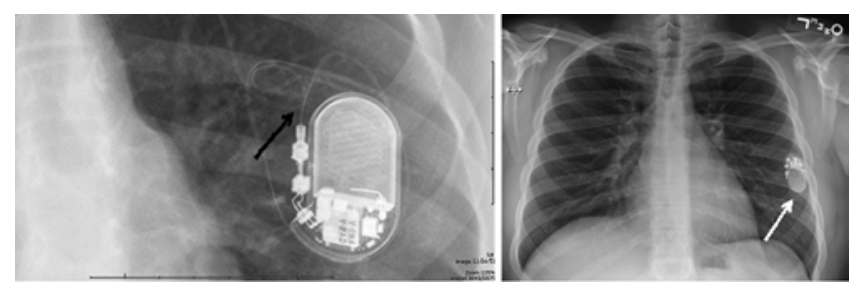

FIG. 1. Preoperative anteroposterior chest radiographs obtained in 2 patients with high lead impedance $(>10,000 \Omega)$, demonstrating discontinuity of the electrode wire (black arrow) and disconnection with coiling in the pocket (white arrow). 
TABLE 2. Impedance and follow-up

\begin{tabular}{ccccc}
\hline $\begin{array}{c}\text { Case } \\
\text { No. }\end{array}$ & $\begin{array}{c}\text { Preop } \\
\text { Impedance } \\
(\Omega)\end{array}$ & $\begin{array}{c}\text { Postop } \\
\text { Impedance } \\
(\Omega)\end{array}$ & $\begin{array}{c}\text { Most Recent } \\
\text { Impedance } \\
(\Omega)\end{array}$ & $\begin{array}{c}\text { Clinical } \\
\text { Follow-Up } \\
(\text { mos })\end{array}$ \\
\hline 1 & Low & 2070 & 1064 & 87 \\
\hline 2 & $<600$ & 2189 & 986 & 53 \\
\hline 3 & Low & 2210 & 2110 & 54 \\
\hline 4 & 681 & 2305 & 1216 & 52 \\
\hline 5 & 1500 & 1528 & 1528 & 12 \\
\hline 6 & Low & 1739 & 1739 & 12 \\
\hline
\end{tabular}

\section{Results}

Follow-up for these patients ranged from 12 to 87 months. All 6 patients have maintained unchanged seizure control. The patients in Cases 3 and 4 underwent revision for IPG battery replacement at the end of the battery's life subsequent to their lead repairs. Both patients remain with the original repaired leads and unchanged appropriate lead impedance. None of these 6 patients experienced infection, vocal cord palsy, or other complications.

\section{Discussion}

Insertion of a VNS has previously been demonstrated to significantly decrease seizures in at least $50 \%$ of patients. ${ }^{4}$ However, device insertion carries inherent risk, including hardware failure and infection necessitating removal or replacement. Battery end of life, lead breakage, and infection are the most common reasons for reoperation. ${ }^{4,6}$ Battery replacement surgeries are expected due to the nonrechargeable nature of the generators and have been associated with a slight, but not statistically significant, increase in infection. In a study by Kahlow and Olivecrona with 143 patients, 3.5\% had superficial infections and $3.5 \%$ had deeper infections requiring removal of the VNS. Rates of infection showed a tendency to increase with each revision. ${ }^{3}$

Lead failure can be attributed to fracture of the lead insulation as well as "micro-injuries" without visible damage. Dlouhy et al. reported on 24 patients who required lead replacement and found that $89 \%$ had no visible fracture of the leads. Their review showed no definitive increase in vascular injuries; however, they had significantly increased mean operating times: 94 minutes (range 53195 minutes) for the initial lead placement of 15 patients included in their study compared with an average duration of 173 minutes (range 108-273 minutes) for the 24 revision surgeries. ${ }^{1}$

Electrode replacement requires exposure of the vagus nerve in the neck and places the carotid artery and internal jugular vein at increased risk of injury. Several studies have been compiled with an overall incidence of lead breakage in VNSs ranging from $0.5 \%$ to $20.8 \% .^{3,5,6}$ In the study by Kahlow and Olivecrona of 143 patients, $11.9 \%$ experienced lead breakage requiring reoperation for lead replacement. ${ }^{3}$ Vascular injury during primary lead insertion occurred in $0.7 \%$, compared with $8.3 \%$ of revision surgeries. ${ }^{3}$

Direct injury to the vagus nerve is another risk of re-
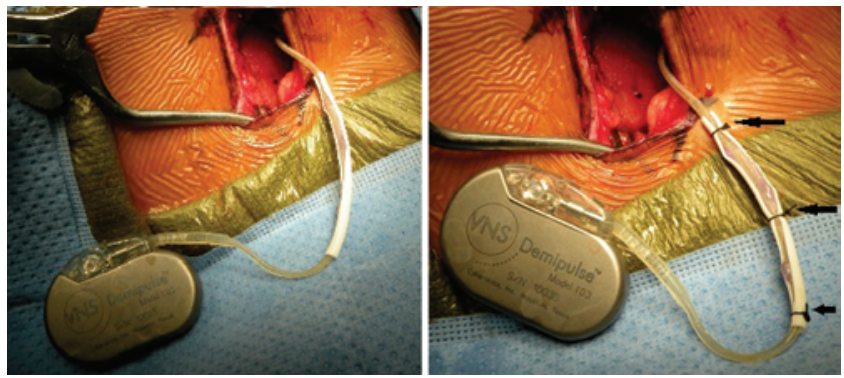

FIG. 2. Left: Side-slit Silastic tubing surrounds the damaged outer sheath. Right: Circumferential silk ties, indicated by arrows, secure the catheter after introduction of the cyanoacrylate glue. Figure is available in color online only.

moving the electrodes. In a separate study, 2 of 36 patients experienced lead fracture and underwent lead replacement, in one case by unwinding the coils from the nerve and in the second case by cutting the coils and removing in pieces. Temporary vocal cord paralysis was observed in the patient whose coils were removed by unwinding. ${ }^{5}$

Despite the increased risk with revision for lead replacement, retained functionality of the stimulator with decreased seizure burden remains a much-desired goal. Dlouhy et al. demonstrated seizure control after replacement to be comparable to that of the primary insertion. ${ }^{1}$ Although our cohort is smaller, we have observed similar seizure control in those patients in whom we repaired the electrodes. This functionality remained over several years. These patients, who have undergone our lead repair technique in lieu of replacement, will require ongoing monitoring to assess the durability of the repaired leads. However, to date, both the efficacy and durability of this repair technique appear to be excellent.

\section{Conclusions}

VNS lead malfunction is a significant complication with resultant increased risk of vascular and nervous injuries during revision and replacement. High lead impedance should trigger further investigation for lead disconnection or discontinuity, whereas low lead impedance may suggest a potential opportunity for lead repair. Repair for lead malfunction due to loss of insulation integrity is an option that obviates the need for reoperation on the neck, reducing the risks of the revision. If proven durable for ongoing use of the VNS, this approach will be of great value for patients with this type of VNS lead malfunction.

\section{References}

1. Dlouhy BJ, Viljoen SV, Kung DK, Vogel TW, Granner MA, Howard MA III, et al: Vagus nerve stimulation after lead revision. Neurosurg Focus 32(3):E11, 2012

2. Hauptman JS, Mathern GW: Vagal nerve stimulation for pharmacoresistant epilepsy in children. Surg Neurol Int 3 (Suppl 4):S269-S274, 2012

3. Kahlow H, Olivecrona M: Complications of vagal nerve stimulation for drug-resistant epilepsy: a single center longitudinal study of 143 patients. Seizure 22:827-833, 2013

4. Morris GL III, Gloss D, Buchhalter J, Mack KJ, Nickels K, Harden C: Evidence-based guideline update: vagus nerve stimulation for the treatment of epilepsy: report of the Guide- 
line Development Subcommittee of the American Academy of Neurology. Neurology 81:1453-1459, 2013

5. Rychlicki F, Zamponi N, Cesaroni E, Corpaci L, Trignani R, Ducati A, et al: Complications of vagal nerve stimulation for epilepsy in children. Neurosurg Rev 29:103-107, 2006

6. Smyth MD, Tubbs RS, Bebin EM, Grabb PA, Blount JP: Complications of chronic vagus nerve stimulation for epilepsy in children. J Neurosurg 99:500-503, 2003

\section{Disclosures}

Dr. Kohrman states that he had a nonfinancial relationship with Cyberonics (research studies and speaker).

\section{Author Contributions}

Conception and design: Ralston, Frim. Acquisition of data: Ralston, Ogden. Analysis and interpretation of data: Ralston, Ogden. Drafting the article: Ralston. Critically revising the article: Ralston, Frim. Reviewed submitted version of manuscript: Ralston, Frim. Administrative/technical/material support: Ogden, Kohrman, Frim. Study supervision: Kohrman, Frim.

\section{Correspondence}

Ashley Ralston, Section of Neurosurgery, The University of Chicago, 5841 S Maryland Ave., MC 3026, Chicago, IL 60637. email: ashley.ralston@uchospitals.edu. 